\title{
Métalepse et image de soi de l'auteur dans le récit de fiction
}

Metalepsis and the author's self-image in fictional narrative

\section{Michèle Bokobza Kahan}

\section{(2) OpenEdition}

Journals

Édition électronique

URL : http://journals.openedition.org/aad/671

DOI : 10.4000/aad.671

ISSN : 1565-8961

Éditeur

Université de Tel-Aviv

Référence électronique

Michèle Bokobza Kahan, « Métalepse et image de soi de l'auteur dans le récit de fiction »,

Argumentation et Analyse du Discours [En ligne], 3 | 2009, mis en ligne le 15 octobre 2009, consulté le

10 décembre 2020. URL : http://journals.openedition.org/aad/671; DOI : https://doi.org/10.4000/aad. 671

Ce document a été généré automatiquement le 10 décembre 2020.

\section{c) (†) $९$}

Argumentation \& analyse du discours est mis à disposition selon les termes de la licence Creative Commons Attribution - Pas d'Utilisation Commerciale - Pas de Modification 4.0 International. 


\title{
Métalepse et image de soi de l'auteur dans le récit de fiction
}

\author{
Metalepsis and the author's self-image in fictional narrative
}

Michèle Bokobza Kahan

1 La problématique articulation du monde réel auquel appartient l'auteur sur le monde fictionnel qu'il invente et à partir duquel nous voulons cerner son image de soi constitue le point de départ d'une réflexion qui tend à préciser les points de jointure des deux niveaux en mettant l'accent sur le texte. La spécificité et la difficulté du récit de fiction par rapport à tous les autres types d'échanges verbaux réside dans l'appartenance des interlocuteurs de l'échange littéraire à des mondes référentiels différents. C'est sur ce point précis que se pose la question: comment parler d'un auteur réel en nous référant à un texte fictionnel? Notre objectif vise à suggérer une piste méthodologique permettant d'appréhender l'image de soi ou l'ethos discursif de l'auteur dans le texte de fiction qu'il produit.

2 Les notions d'« auteur implicite » et de «narrateur non fiable » inaugurées par Booth, puis reprises et retravaillées par la critique narratologique et la poétique, privilégient le texte de fiction et ses fonctionnements internes tout en permettant de dégager la présence diffuse de l'auteur dans la fiction, de mieux cerner les procédés d'évitement, de distribution, de diffraction, d'exhibition, et de saisir les lieux où l'auteur signale sa présence et suggère son intervention. Néanmoins, la prise en compte de la perméabilité des frontières entre l'intérieur et l'extérieur du texte m'invite à concilier des concepts narratologiques classiques avec la notion d'«ethos discursif " qui ouvre le texte en amont, versant auteur. L'analyse du discours définit l'ethos comme une image verbale construite dans le discours par celui qui prend la parole pour mener son audience vers une adhésion et un accord (Amossy 1999; 2006 [2000]). Admettre la présence d'une instance énonciatrice première (instance auctoriale) se référant à l'auteur réel implique nécessairement qu'il y a construction d'un ethos dans le discours de fiction, ce qu'Amossy appelle ici même un "ethos auctorial». La complexité du dispositif énonciatif particulier de la fiction et la dissémination de l'image de soi de l'auteur dans de multiples voix actorielles renvoient une fois de plus à la difficile articulation de deux 
registres narratifs différents. C'est précisément sur ce point que le choix de la métalepse trouve sa pertinence.

La métalepse déclenche un mécanisme narratif qui transforme l'espace discursif en un lieu d'accueil privilégié de l'«auteur réel», que je mets ici entre guillemets pour rappeler la complexité de la notion en littérature. Parce qu'elle consiste à produire un effet de réel dans la fiction par l'intrusion d'instances extradiégétiques dans l'univers intradiégétique et/ou par l'intervention de personnages intradiégétiques au niveau extradiégétique, la métalepse déstabilise la fonction ludique inhérente au roman en brouillant les frontières qui séparent les instances narratives de part et d'autre du dispositif énonciatif. Dès lors que les instances énonciatives qui se situent en dehors de l'espace narratif fictif (auteur, lecteur) se mêlent aux instances narratives fictives, ou que des narrateurs extradiégétiques censés rapporter le discours de narrateurs seconds s'introduisent dans des histoires auxquelles ils n'appartiennent pas, ou encore que des personnages de la fiction sortent de leur cadre pour envahir l'univers du narrateur, la transition d'un niveau narratif à un autre apparaitt comme transgressive (Genette 1972 : 2004).

Les notions de Booth mettent justement en valeur la relation dynamique forte qui existe entre les agents du dispositif énonciatif de part et d'autre du récit. Elles se sont enracinées dans le langage de la critique littéraire parce qu'elles concernent les rapports compliqués, mais néanmoins cruciaux, qui se tissent entre l'auteur, l'oeuvre et ses composantes énonciatives, et le lecteur. Pour Booth, l'auteur implicite désigne l'instance suprême qui oriente le sens et les enjeux du texte. Sa présence conditionne les propriétés constitutives d'une image d'auteur spécifique :

[...] il est clair que l'image que reçoit le lecteur de cette présence est un des effets les plus importants voulus par l'auteur. Quelque impersonnel qu'il tente d'être, son lecteur se construira inévitablement une image du scribe officiel qui écrit de cette manière, et bien entendu, ce scribe officiel ne sera jamais neutre face à toute valeur (Booth 1961, trad. Brun, 2001 : 192).

5 C'est par rapport à cette première notion que le terme de "narrateur non fiable » émerge. Il s'agit du narrateur dont la relation et/ou l'interprétation des faits, des événements, des personnages et de leurs pensées, y compris les siennes, est soit lacunaire soit déficiente, soit autre que ce que dit ou laisse entendre l'auteur implicite (Chatman 1990 ; Lanser 2001; Yacobi 2001 ; Nünning 2005). Cet écart participe par voie de négation à la construction de l'identité de l'auteur implicite, ce second moi textuel qui renvoie, malgré divers degrés de distinction envisageables (Chatman 1978; Ginsburg et Rimmon-Kenan 1999), au responsable premier du texte, l'auteur réel, " mort et ressuscité », pour reprendre le titre évocateur de Burke (1992). Notons qu'en 1952, lorsque Booth parle du "narrateur conscient de soi ", il distingue déjà l'instance narratrice du récit de l'instance auctoriale implicite qui se trouve à la fois dans et en dehors du texte: " Nearly everyone has given up the once popular belief that Laurence Sterne's writing methods are as chaotic as those of Tristam Shandy. Although Tristam depended on Almighty God for his next move, Sterne, most reputable critics now believe, depended on a reasonably elaborate plan » (Booth 1952 : 163).

Booth délimite son champ d'investigation à l'espace discursif de la fiction, sans ignorer pour autant le rapport de réciprocité permanent et dynamique entre texte et horstexte. L'auteur implicite et ses narrateurs fiables ou non fiables donnent à voir la dimension communicationnelle du texte de fiction et pas seulement à l'intérieur du monde inventé (la diégèse): "How could anyone ever believe that the author's 
intentions about a work are irrelevant to how we read it? » s'exclame Booth, fidèle à lui-même, dans son tout dernier article sur la question de l'auteur implicite (2005: 75). Comme tout acte de langage, la littérature est un acte de communication, et en tant que tel, elle induit un échange entre au moins deux instances interlocutrices. Aussi nous tournons-nous de gré ou de force vers l'origine première du texte, qui est, quoiqu'en dise Barthes (1967), l'auteur. Sans le confondre avec la personne " en chair et en os ", l'auteur empirique, producteur d'un texte, existe toujours et doit être pris en compte. Sa dilution fonctionnelle dans la structure du texte (Foucault 1969), son abstraction psychologique dans le discours littéraire fictionnel (Couturier 1995), ou son expulsion de l'analyse narratologique (Genette 1972) ne peuvent l'empêcher de vivre dans le texte contre vents et marées (Briosi 1986 ; Maingueneau 2004 ; Booth 2005).

7 Parallèlement, les études consacrées à la métalepse (Malina 2002; Genette 2004 ; Pier et Schaeffer [éds] 2005) corroborent la nécessaire prise en compte du sujet-auteur. Elles montrent comment la métalepse souligne dans le récit la spécificité de la relation causale qui «unit l'auteur à son œuvre, le producteur d'une représentation à cette représentation elle-même » (Genette 2005 : 25). Cette propriété métatextuelle confirme la présence d'un énonciateur premier (auteur implicite) se situant à la fois en dehors du récit et dans le récit, et se positionnant par rapport à lui. La transition métaleptique désigne aussi le passage du monde de la narration au monde des événements narrés et vice versa. De plus, la non fiabilité du narrateur peut se construire par le biais de transgressions narratives comme la métalepse (Kindt 2005, Pier et Schaeffer [éds]). Le procédé a donc l'avantage de faire entendre la voix de l'auteur implicite, instance extradiégétique qui se tapit à l'ombre du narrateur considéré comme personnage sur le plan discursif, une voix que certains n'hésiteraient pas à appeler Scarron, Furetière, Bordelon ou Mouhy, pour ne citer que les auteurs dont il sera question ici.

8 Les effets de trouble que la métalepse produit dans la distinction des niveaux narratifs distincts égratignent le caractère imaginaire de la fiction et établissent un lien de connivence avec le lecteur réel. C'est au regard de la conception aristotélicienne de la mimesis que s'opère la transgression métaleptique, une conception qui constitue

le principal fondement de la notion somme toute complexe de pacte narratif qui, à un travail d'écriture soucieux d'édifier une image plausible du réel sans pour autant se réduire à sa reproduction servile, fait correspondre une attitude lectorale symétrique et homogène, reposant sur une mise entre parenthèses intentionnelle et provisoire de l'incrédulité (Wagner $2002: 237$ ).

9 Si la métalepse au plan narratif manifeste le passage transgressif d'un niveau à un autre, au plan de la représentation, elle déstabilise le fonctionnement représentationnel et pose le problème de la référence. Elle transforme le contrat de lecture initialement fondé sur un principe de vraisemblance et le situe autour d'un savoir partagé de l'illusion. L'exemple le plus connu pour la littérature du $18^{\mathrm{e}}$ siècle demeure sans doute celui de Diderot qui, dans Jacques le Fataliste, met en évidence l'invention de l'histoire par l'auteur.

10 Pour toutes ces raisons, la métalepse produit un discours qui ressemble à ceux des « seuils» textuels comme la note, la préface, la dédicace qui favorisent la rencontre, le frottement voire la confusion des mondes réel et fictif (Genette 1987; Herman 1998; Bokobza Kahan 2004, 2009). Inévitablement, elle soulève la question de l'auteur dans le texte, un auteur implicite dont la manifestation, les composantes et les enjeux inscrits dans les mécanismes narratifs métaleptiques participent de la construction d'un ethos qui est, selon nous, celui de l'auteur réel. En effet, même s'il perd dans la fiction toute 
possibilité d'énonciation explicitement assumée, celui-ci demeure malgré tout l'instance souveraine qui décide de cacher ou d'exhiber les coulisses de la fiction. Les combinaisons, les ruptures et les intrusions énonciatives et narratives effectuées dans le texte résultent de ses choix personnels. Lui seul décide de la porosité ou au contraire de l'étanchéité des frontières qui séparent les mondes de la fiction et de la réalité. C'est encore lui qui choisit de s'éclipser du récit ou qui prétend s'y immiscer. La présence ou l'absence auctoriale, la confusion des mondes narré et narrant, les transgressions narratives, sont les procédés constitutifs du rapport que l'auteur construit avec le lecteur, de sa conception de la littérature en général et de sa propre pratique d'écriture en particulier. Parce c'est l'auteur qui s'adresse au lecteur en sa qualité de créateur des personnages et d'organisateur du récit, et non le narrateur qui raconte l'histoire des personnages comme s'ils existaient vraiment, nous sommes avec la métalepse essentiellement confrontés à une interrogation sur le positionnement de l'auteur par rapport à son œuvre et au lecteur pour qui il écrit. Or, c'est bien dans ce rapport que s'élabore en partie l'image de soi de l'auteur dans son texte.

Les exemples qui suivent permettront de montrer comment différents types de métalepse véhiculent des enjeux littéraires différents corollaires d'une image d'auteur à la fois inscrite dans le texte de fiction et reliée à un positionnement dans le champ littéraire et à un ethos préalable spécifique (Amossy et Hadad 1999, Amossy [éd.]). Les multiples interventions d'auteur dans la fiction provoquent les effets les plus divers, voire des effets contraires, selon le degré de transgression à l'aune duquel il est possible d'apprécier le caractère autoritaire ou ludique, conventionnel ou subversif, sérieux ou ironique, traditionnel ou contestataire, conscient ou inconscient, ostentatoire, péremptoire, conciliant, complaisant et/ou divertissant de l'auteur dans son texte.

Tout en m'appuyant sur des critères formels et fonctionnels déjà existants comme la métalepse de régie (Genette 2004 ; Cohn 2005, Pier et Schaeffer [éds]), la métalepse rhétorique (Ryan 2005, Pier et Schaeffer [éds]), ou la métalepse ontologique (Nelles 1997), je propose ici des distinctions qualificatives d'ordre générique comme la métalepse ludique, la métalepse pamphlétaire, la métalepse syncrétique et la métalepse libertaire. Ces distinctions permettent de mieux dégager le rapport corrélatif entre un type d'ingérence narrative et une manière d'être dans le texte qui renvoie à l'ethos auctorial. Les quelques cas de figure choisis illustrent également l'extraordinaire richesse et modernité d'écrivains marginaux situés entre Cervantès et Sterne dans la France des $17^{\mathrm{e}}$ et $18^{\mathrm{e}}$ siècles.

\section{La métalepse ludique}

13 La «métalepse de régie » relève d'un procédé narratif qui met en valeur l'importance du rôle d'organisateur et de gestionnaire rempli par l'instance narrative auctoriale. Au $17^{\mathrm{e}}$ siècle, Le roman comique (1651) de Scarron constitue l'exemple emblématique de ce procédé narratif. L'auteur régisseur fait des apparitions soudaines où il s'autoreprésente dans son activité d'écrivain en train d'écrire le livre que nous lisons :

[...] par une générosité inouïe en une maîtresse de tripot, [elle] permit au charretier de faire manger ses bêtes tout leur soûl. Il accepta l'offre qu'elle lui fit et, cependant que ses bêtes mangèrent, l'auteur se reposa quelque temps et se mit à songer à ce qu'il dirait dans le second chapitre (Scarron 1981 [1651] : 67). 
Là-dessus deux hommes entrèrent dans le logis, qui furent reconnus par notre comédien pour être de ses camarades, desquels nous parlerons plus amplement au suivant chapitre (ibid. : 81 ). effets contradictoires que provoque la malléabilité de l'auteur sont tous reliés à l'image
de soi auctoriale dans le texte. Maîtrise et impuissance, savoir et ignorance : l'auteur se
trouve souvent dépassé par sa propre création, mais une fois dépossédé de son œuvre,
il opte pour le rire et le burlesque comme en témoigne la métalepse qui ouvre le
chapitre 12 :

Je suis trop homme d'honneur pour n'avertir pas le lecteur bénévole que, s'il est scandalisé de toutes les badineries qu'il a vues jusques ici dans le présent livre, il fera fort bien de n'en lire pas davantage ; car en conscience il n'y verra pas d'autre chose, quand le livre serait aussi gros que le Cyrus; et si, par ce qu'il a déjà vu, il a de la peine à se douter de ce qu'il verra, peut-être que j'en suis logé là aussi bien que lui, qu'un chapitre attire l'autre et que je fais dans mon livre comme ceux qui mettent la bride sur le col de leurs chevaux et les laissent aller sur leur bonne foi. Peut-être aussi que j'ai un dessein arrêté et que, sans emplir mon livre d'exemples à imiter, par des peintures d'actions et de choses tantôt ridicules, tantôt blâmables, j'instruirai en divertissant de la même façon qu'un ivrogne donne de l'aversion pour son vice et peut quelquefois donner du plaisir par les impertinences que lui fait faire son ivrognerie. Finissons la moralité et reprenons nos comédiens que nous avons laissés dans l'hôtellerie (ibid.: 111).

L'incertitude de l'auteur, démiurge déchu comparé à un ivrogne, équivaut à l'ignorance $\mathrm{du}$ lecteur à qui l'on ne peut rien promettre. Tous deux attendent avec la même curiosité la suite des événements, tous deux observent le produit dont ils sont les coresponsables par le double acte de l'écriture et de la lecture sans pouvoir s'en emparer. Le livre loin de leur appartenir se construit presque contre leur gré. Leur sort commun 
accentue la dimension communicationnelle du discours littéraire. Le rapport égalitaire qui se dégage de la dynamique produite par les métalepses remplit plusieurs fonctions. En premier lieu, il renforce la désinvolture affichée de l'auteur : «J'ai fait le précédent chapitre un peu court, peut-être que celui-ci sera plus long; je n'en suis pourtant pas bien assuré, nous allons voir » (ibid. : 167) ; ensuite, il souligne son refus catégorique du sérieux et sa défiance ironique envers le romanesque outré propre au roman héroïcogalant; il caractérise enfin le ton comique et anti-solennel du roman: "Ceux qui auront eu assez de temps à perdre pour l'avoir employé à lire les chapitres précédents doivent savoir, s'ils ne l'ont pas oublié, que le curé de Domfront était dans l'un des brancards qui se trouvèrent quatre de compagnie dans un petit village, par une rencontre qui ne s'était peut-être jamais faite " (134). Le jeu métaleptique permet à l'auteur comique de surprendre le lecteur pour l'amuser, de l'inviter à railler les modèles classiques sérieux.

Ces éléments nous renvoient à l'image d'auteur que construit Scarron ailleurs, dans ses nombreuses œuvres burlesques et ses nouvelles tragi-comiques ainsi que dans des discours péritextuels étonnants comme la succulente dédicace «Au Lecteur qui ne m’a jamais vu » qui ouvre le Roman comique:

Lecteur, qui ne m'as jamais vu et qui peut-être ne t'en soucies guère, à cause qu'il n'y a pas beaucoup à profiter à la vue d'une personne faite comme moi, sache que je ne me soucierais pas aussi que tu me visses si je n'avais appris que quelques beaux esprits facétieux se réjouissent aux dépens du misérable et me dépeignent d'une autre façon que je suis fait. [...] Au défaut de la peinture, je m'en vais te dire à peu près comme je suis fait. J'ai trente ans passés, comme tu vois au dos de ma chaise. Si je vais jusqu'à quarante, j'ajouterai bien des maux à ceux que j'ai déjà soufferts depuis huit ou neuf ans. J'ai eu la taille bien faite, quoique petite. Ma maladie l'a raccourcie d'un bon pied. Ma tête est un peu grosse pour ma taille. J'ai le visage assez plein, pour avoir le corps très décharné ; des cheveux assez pour ne porter point de perruque ; j'en ai beaucoup de blancs en dépit du proverbe. [...] Mes jambes et mes cuisses ont fait premièrement un angle obtus, et puis un angle égal, et enfin un aigu. Mes cuisses et mon corps en font un autre et, ma tête se penchant sur mon estomac, je ne représente pas mal un Z. J'ai les bras raccourcis aussi bien que les jambes, et les doigts aussi bien que les bras. Enfin, je suis un raccourci de la misère humaine. Voilà à peu près comme je suis fait. Puisque je suis en si beau chemin, je te vais apprendre quelque chose de mon humeur ; [...] J'ai toujours été un peu colère, un peu gourmand et un peu paresseux. J'appelle souvent mon valet sot et un peu après Monsieur. Je ne hais personne; Dieu veuille qu'on me traite de même. Je suis bien aise quand j'ai de l'argent, et serais encore plus aise si j'avais la santé. Je me réjouis assez en compagnie; je suis assez content quand je suis seul. Je supporte mes maux assez patiemment; et il me semble que mon avant-propos est assez long et qu'il est temps que je finisse.

19 Ce texte qui mériterait une analyse plus détaillée offre l'autoportrait de l'auteur réel, le Scarron malade et déformé, bouffon talentueux et provocateur. La présentation concilie l'aspect grotesque du paralytique, le franc parler du satiriste qui n'a pas peur de se prendre pour cible et la bonne humeur de l'auteur comique. La manière directe, voire brutale dont il met en scène son moi empirique dans le péritexte diffère de l'image de soi qui se dégage subtilement des métalepses. Alors que l'adresse directe au lecteur l'amène à concrétiser dans les mots les tares physiques et la condition de paralytique, la fiction lui permet de dessiner en pointillé à travers les transgressions narratives relevées l'image d'un auteur souple et habile, léger comme une plume, apparaissant et disparaissant au gré des aventures des héros de papier. Cette 
complémentarité complexe fort intéressante corrobore l'idée d'une imbrication d'images de soi auctoriales qui renvoient à la figure de l'auteur signataire de l'œuvre.

La métalepse marque dans le discours la puissance de rire étonnante de l'auteur satirique qu'est Scarron. Espace privilégié de la mise en scène de l'auteur au travail, la métalepse confirme aussi l'importance de l'acte de narrer. Or, toute la force de Scarron, auteur empirique, cul-de-jatte cloué sur une chaise, réside dans l'art de subjuguer son auditoire en lui contant des histoires : " jamais homme n'a mieux entendu le style et le caractère de la narration ", écrit Huet dans De l'origine des romans à propos de Scarron (Giraud 1981: 29). Rappelons que la parole du conteur se déploie dans l'oralité dont la spécificité réside dans l'immédiat, le spontané et l'imprévisible. La métalepse récupère les caractéristiques de l'image d'auteur dans le champ littéraire pour les retravailler dans la fiction. Lieu d'un échange divertissant instauré entre l'auteur et son lecteur, elle laisse voir l'image d'un auteur comique, d'un conteur plaisant et moqueur, d'un prestidigitateur qui change d'identité et de monde en un tour de passe. Ce n'est que dans un second temps que la métalepse constitue une forme d'engagement critique sur la fiction et sa poétique, même si ce positionnement coïncide parfaitement avec l'auteur de poésie satirique et de théâtre qu'est Scarron.

\section{La métalepse pamphlétaire}

21 Chez Furetière, auteur un peu plus tardif $d u 7^{\mathrm{e}}$ siècle, le procédé métaleptique prend une dimension critique ferme et univoque qui se substitue à la tonalité ludique que lui donne son prédécesseur. L'interventionnisme auctorial observé auparavant chez Scarron vise, dans Le roman bourgeois (1666) de Furetière, à inscrire dans le discours la prise de distance de l'auteur à l'égard de sa production littéraire, voire à exprimer une désolidarisation. Par sa fréquence et son envergure spatiale, la métalepse troue le tissu diégétique, faisant ainsi obstacle à toute participation mimétique du lecteur pour laisser entrevoir un autre discours, polémique et sérieux, sur le roman en général et sur certaines pratiques d'écriture en particulier :

Je me doute bien qu'il n'y aura pas un lecteur (tant soit-il bénévole) qui ne dise icy en luy-même : «Voicy un méchant Romaniste! Cette histoire n'est pas fort longue ny fort intriguée. Comment ! il conclud d'abord un mariage, et on n'a pas coûtume de les faire qu'à la fin du dixième tome! ». Mais il me pardonnera, s'il luy plaist, si j'abrège et si je cours en poste à la conclusion. Il me doit mesme avoir beaucoup d'obligation de ce que je le guéris de cette impatience qu'ont beaucoup de lecteurs de voir durer si long-temps une histoire amoureuse, sans pouvoir deviner quelle en sera la fin. Néantmoins, s'il est d'humeur patiente, il peut sçavoir qu'il arrive, comme on dit, beaucoup de choses entre la bouche et le verre. Ce mariage n'est pas si avancé qu'on diroit bien et qu'il se l'imagine. (Furetière 1666 : 17)

Ou encore cette longue diatribe contre les romans héroïco-galants dont je ne cite que quelques lignes :

Je ne tiens pas necessaire de vous rapporter icy par le menu tous les sentiments passionnez qu'il estalla et toutes les raisons qu'il allegua pour l'y faire resoudre, non plus que les honnestes resistances qu'y fit Javotte, et les combats de l'amour et de l'honneur que se firent dans son esprit: car vous n'estes gueres versez dans la lecture des romans, ou vous devez sçavoir 20 ou 30 de ces entretiens par cœur, pour peu que vous ayez de memoire. Ils sont si communs que j'ay veu des gens qui, pour marquer l'endroit où ils en estoient d'une histoire, disoient : J'en suis au huictiesme enlevement, au lieu de dire : J'en suis au huictiesme tome. Encore n'y a-t'il que les 
autheurs bien discrets qui en fassent si peu, car il y en a qui non seulement à chaque tome, mais à chaque livre, à chaque episode ou historiette, ne manquent jamais d'en faire. Un plus grand orateur ou poëte que moy, quelque inventif qu'il fust, ne vous pourroit rien faire lire que vous n'eussiez veu cent fois (ibid. : 136). coïncide avec un dialogue entre l'auteur dans le texte et son lecteur extérieur ne se contente pas de rompre de manière ponctuelle l'illusion de la fiction comme c'était le cas chez Scarron. La fréquence des interruptions, leur longueur, leur caractère à la fois polémique et ironique dote l'ingérence métaleptique d'un degré d'ostentation qui nous éloigne de la métalepse de régie. La visée organisatrice qui caractérise celle-ci disparaît au profit d'une dé-liaison du texte, de sa fragmentation, d'une cassure irréparable de l'effet illusionniste de la fiction. Ce désengagement de l'auteur à l'égard de son récit implique une prise de conscience qui participe en retour de son engagement intellectuel contre ce «mal-heureux siècle où on ne s'amuse qu'à la bagatelle » (ibid. : 84). La dimension satirique très prononcée de la métalepse métafictionnelle souligne ici le rapport conflictuel que l'auteur entretient avec les divers agents du champ littéraire de l'époque : les auteurs, les éditeurs, les lecteurs et leur horizon d'attente, les genres littéraires. situation paradoxale où se trouve l'auteur implicite. Pratiquer et critiquer dans un même temps l'écriture romanesque influe inévitablement sur une œuvre qui a été d'ailleurs méconnue et incomprise par ses contemporains. Les mécanismes narratifs métaleptiques utilisés qui permettent de dégager une voix auctoriale distincte de celle du narrateur contribuent à la construction de l'ethos auctorial qui se trouve lui-même en rapport avec des images d'auteur extérieures à la fiction.

Le succès de sa Nouvelle Allégorique ou Histoire des derniers troubles arrivés au royaume d'Eloquence (1658) place Furetière, habitué des cercles littéraires parisiens. parmi les satiristes en vogue. En y décrivant par l'allégorique et le satirique l'histoire de la littérature de 1630 à 1658, il fait preuve d'un savoir et d'une pensée théorique sur la poétique et le langage qui ne se dément pas lorsque quelques années plus tard il compose avec Boileau, Racine et d'autres, la célèbre parodie du Cid, Le chapelain décoiffé. Auteur satirique qui entend exploiter, à l'instar de ses amis, la veine réaliste pour combattre la littérature romanesque héroïco-galante et idéaliste, Furetière est considéré comme un auteur engagé. A ses yeux, l'ineptie des interminables romans à clef de Scudéry mène à la médiocrité de la pensée et au galimatias rhétorique, une position qui est reliée au travail passionné d'érudition et de lecture qu'il a mené pendant de longues années en tant que membre de l'Académie Française en vue de la publication du Dictionnaire de la langue française. Sur ce point également, Furetière se démarque en accusant de lenteur, de fainéantise voire d'incapacité ses collègues académiciens. Lorsqu'il décide de faire cavalier seul, il devient l'objet d'une cabale impitoyable qui aboutit à son expulsion infâmante de l'Académie. La rédaction du Dictionnaire universel et sa publication posthume immortalisent son nom, douce vengeance bien méritée.

Lorsqu'il écrit Le Roman Bourgeois en 1666, Furetière est un auteur reconnu, réputé, membre de l'Académie depuis 1662, ami des auteurs les plus célèbres du siècle. En même temps, il se positionne fortement contre certaines tendances littéraires, travaille de préférence en solitaire et joue la carte du combattant avant-gardiste. Parfaitement introduit dans le milieu littéraire de son époque, à la fois passionné et

Argumentation et Analyse du Discours, 3 | 2009 
carriériste, Furetière ne se contente pas de réfléchir sur les genres en vogue et participer activement aux débats littéraires de son temps. Il se bat avec obstination et âpreté sur des sujets qui lui tiennent à coeur.

Dans son roman, la manière dont il casse le pacte de l'illusion fictionnelle et fait obstacle à toute possibilité d'identification, le degré de distanciation à l'égard du récit et des personnages pour qui il n'exprime aucune sympathie, l'ironie critique qui loin de provoquer le rire invite plutôt au mépris, constituent les composantes de la métalepse pamphlétaire. Une métalepse qui participe de la construction de l'ethos à la fois savant et satiriste, intimement engagé dans une cabale contre la gent des lettres, distant et provocateur.

\section{La métalepse syncrétique}

L'écriture contestataire qui caractérise l'œuvre étrange de Bordelon (1653-1730), contemporain de Furetière, se développe essentiellement entre 1697 et 1715. La métalepse joue un rôle prépondérant dans le schéma narratif de son œuvre. Par sa fréquence et sa présence, elle ressemble à celle de Furetière. A l'instar de ce dernier, elle est également en prise sur l'actualité littéraire encore brûlante de la querelle des Anciens et des Modernes et participe d'une dénonciation globale des fictions comme en témoigne par exemple la préface de l'Histoire des imaginations extravagantes de Monsieur Oufle. L'auteur y prétend élargir le programme de Cervantès en englobant dans sa dénonciation de la fiction tous les écrits, même ceux chargés de transmettre un savoir présenté comme sérieux. Cependant, au-delà de cette première fonction métafictionnelle, la métalepse chez Bordelon se détache de la métalepse pamphlétaire évoquée plus haut non seulement par son ton moins militant, mais surtout parce qu'elle se replie à bien des égards sur l'auteur et son univers. Elle condense les ambiguïtés de la position de l'auteur et souligne une relation causale très particulière qui unit cette fois l'auteur à son œuvre.

Dans un premier temps, Bordelon semble procéder lui aussi à un interventionnisme qui correspond à la fonction anti-illusionniste de la métalepse sans constituer véritablement une remise en cause des conventions de l'art de conter. Comme chez Scarron, il suspend le récit pour laisser la place aux remarques personnelles de son narrateur omniscient. Celui-ci souligne son droit d'ingérence, voire son omnipotence, dans le récit qu'il produit: " Nous avons laissé notre nouveau Lycaon courant les rues, après avoir donné une terrible chasse à la musique nocturne qui s'était trouvée dans son chemin. Passons à ses autres aventures" (Bordelon, Histoire de Monsieur Oufle : 34), "Mais laissons-leur le plaisir de crier victoire après avoir fui de leur mieux; et revenons à M. Oufle » (ibid. : 36); "Avant de passer outre, et de continuer le récit des aventures de M. Oufle, je parlerais succinctement de ceux qui, comme lui, ont l'esprit faible et crédule » (ibid.: 155). Le degré d'intervention massive du narrateur contamine finalement le texte. Sans contrevenir à son statut extradiégétique de narrateur qui surplombe le récit, l'auteur produit un effet d'ambiguïté dans la mesure où il semble écrire dans le monde même de la fiction :

Quoique M. Oufle fût tout essoufflé, tant il parlait avec véhémence, et avec vitesse, il ne laissa pas de continuer. Pour moi, je juge à propos de me donner le temps de respirer moi-même, pour donner le même loisir au lecteur; et ainsi le reste de sa tirade sera pour le chapitre suivant (ibid. : 104). 

d'énonciation et l'univers de l'énoncé provoque deux effets contraires: d'une part, le jeu de la double temporalité de l'histoire et de la narration qui correspond à l'effet antiillusionniste de la métalepse, et d'autre part, la représentation de l'immersion de l'instance auctoriale à l'intérieur de l'univers fictionnel qui renforce l'effet de réel.

31 Cette double fonction contradictoire apparaît de façon encore plus nette dans Gomgan ou l'Homme prodigieux transporté dans l'air, sur la terre et sous les eaux (1711). L'instance d'énonciation de la préface qui renvoie à la figure de l'auteur attribue la paternité du texte à venir à un personnage de papier, le héros éponyme. Mais cette délégation de pouvoir somme toute traditionnelle se trouve déstabilisée par le dialogue qui s'instaure entre l'instance auctoriale et le personnage-narrateur, figure hautement fantastique comme l'atteste le titre: «Si Gomgan, en m'envoyant son manuscrit par ce courrier fugitif dont j'ai parlé, y avait joint quelques preuves pour autoriser ce qu'il avance, j'en ferais part au public d'autant plus volontiers que je les crois nécessaires pour attirer la crédulité » (Bordelon 1711, Herman 1991: 67). Gomgan s'introduit dans l'espace extérieur de la fiction en envoyant la suite de son manuscrit à l'auteur et en lui imposant la manière de rédiger ses aventures. Il surgit dans l'univers de la production du texte pour remplacer son générateur à qui il dicte le texte. L'échange révèle une distribution inattendue des rôles. L'auteur implicite désigne son héros de papier comme le créateur de l'ouvrage au détriment de son porte-parole officiel dans le texte, le narrateur premier.

Leffet d'étrangeté que provoque l'estompage des frontières entre l'univers des personnages et celui de l'auteur engendre un brouillage identitaire de l'instance auctoriale qui bascule du côté de la fiction. L'enchevêtrement des rôles opère un renversement entre réalité et imaginaire qui produit une identification entre l'auteur et son personnage de fiction. La fictionnalisation de l'auteur renforce en retour l'illusion romanesque, et le mouvement cyclique de va-et-vient qui s'ensuit indique le contact permanent et dynamique entre l'auteur et la fiction. La métalepse prend donc en charge une situation paradoxale où l'auteur implicite refuse de trancher entre l'illusionnisme romanesque et la véridicité de la situation d'énonciation auctoriale, refuse de décider qui des deux assume le récit, le narrateur ou le héros du récit.

Au regard de l'interpénétration des mondes extra- et intra-diégétiques, les demandes de confiance à l'adresse du lecteur ne peuvent que dérouter un peu plus ce dernier: "J'ai longtemps délibéré avant que de me déterminer à dire sincèrement de quelle manière ces aventures me sont venues, et cela parce que cette manière n'a rien d'assez considérable pour leur donner plus de poids et les faire mieux recevoir » (ibid.: 66). "Qu'on ne me demande donc pas de plus grands éclaircissements, à moins qu'on ne veuille absolument que j'en invente pour me délivrer des importuns » (ibid.). On sait que le fait de répéter qu'on ne ment pas éveille rapidement la méfiance de celui que l'on veut convaincre. Le procédé freudien de dénégation est connu. L'effet de suspicion que provoquent les trop nombreuses affirmations de l'instance auctoriale signale la dimension parodique du texte alors même que l'auteur dans le texte exige une adhésion à ses dires. Le choix générique de la parodie qui renvoie à l'écrivain producteur du texte indique un positionnement critique et distancié par rapport aux œuvres littéraires parodiées. Le procédé de dénégation relevé révèle par contre une incapacité de la part de l'auteur dans le texte à se déconnecter du monde possible qu'il invente. L'imbrication des niveaux et le lien paradoxal qui les unit complexifie une œuvre

Argumentation et Analyse du Discours, 3 | 2009 
souvent lassante par ses longueurs et ses incohérences. En dénonçant diverses techniques d'illusion, Bordelon ne cherche ni à se distinguer d'une pratique qui tend à imputer le roman à une instance fictive, ni à effacer les références aux conditions réelles de production du texte, ni à se poser en autorité explicitant ses intentions. L'ambiguïté d'une telle position réapparaît ailleurs quand l'instance auctoriale proclame son indifférence à l'éventuel succès ou échec d'un texte qui n'est pas de lui ! (La coterie des anti-façonniers, 1716; Le voyage forcé de Becafort hypocondriaque, 1709). Ce type de désolidarisation à l'égard de sa production romanesque entraîne un effacement de l'autorité auctoriale qui prend, comme on l'a vu, une forme originale dans l'œuvre de Bordelon.

La métalepse ontologique qui, selon Nelles, réunit le monde réel et le monde imaginaire et, ce faisant, jette le doute sur l'existence de frontières certaines entre eux, remplit ici une fonction particulière: elle marque l'omniprésence de l'auteur autant que son absence. Elle inscrit dans le discours l'importance du rôle de l'illusionniste tout en indiquant la répugnance de l'auteur pour le mensonge. De plus, elle signale la pathologie de celui qui ne peut cesser d'écrire dès lors qu'il s'est risqué dans les terres dangereuses de la littérature. C'est dans ce sens qu'il convient de lire des textes où la dénonciation des méfaits de l'écriture coïncide avec une opposition également virulente à la lecture. Toute personne se frottant à la chose littéraire est condamnée à perdre la raison (Histoire des imaginations extravagantes de Monsieur Oufle, 1689, Les malades de belle humeur, ou lettres divertissantes écrites de Chaudray, 1697, Le voyage forcé de Becafort hypocondriaque, 1709).

Le phénomène de réflexivité du roman, dont Bordelon est l'un des représentants (Sermain 2002), amène l'auteur à instaurer un rapport nouveau avec son livre. La dénonciation du romanesque qui définit le modèle de l'antiroman débouche chez Bordelon sur une absorption du récit par la parole qui est censée le porter et, corrélativement, sur la manifestation de plus en plus envahissante de l'activité d'écriture. C'est donc bien vers soi que l'auteur se retourne et c'est de sa propre expérience qu'il nourrit son œuvre, au point d'affirmer dans un ouvrage intitulé $L a$ langue (1707): «J'ai fait ce livre, et ce livre me fait tous les jours ». Interchangeabilité des instances et instabilité du sens produisent une œuvre qui affiche l'absurdité de l'écriture et anéantit le romanesque pour laisser un vide vertigineux dans lequel auteur et personnages sont aspirés vers les airs.

De ce tourbillon discursif se dégage l'image de soi d'un auteur qui en perdant la tête perd également la maîtrise de sa narration, caractéristique qui corrobore l'idée d'une écriture extravagante. Aussi une corrélation forte s'établit-elle entre ce qui se lit dans le texte et ce que nous connaissons de l'écrivain dans le champ littéraire de son temps. L'ethos auctorial fou et prolixe qui coïncide à bien des égards avec l'image de l'auteur implicite instable et capricieux se comprend mieux si l'on se souvient du statut d'écrivain marginal qu'occupe Bordelon. La rareté des indications biographiques concernant le personnage de l'écrivain confirme sa position d' " outsider » qui n'aspire ni à une reconnaissance dans le champ littéraire, ni à un rapport de connivence avec le lecteur. Cet ethos qui se développe dans le champ social de l'époque s'inscrit dans la logique d'une écriture dénonciatrice des écrits, romanesques et sérieux, et de leurs effets pernicieux sur un lecteur naïf, prompt à prendre des vessies pour des lanternes. Néanmoins, la dénonciation demeure incompatible avec le plaisir évident qu'éprouve Bordelon à écrire, à produire des énoncés. Auteur prolixe qui revendique haut et ferme 
le "plaisir» de l'écriture, Bordelon prône simultanément une liberté, voire une indifférence totale, à l'égard des institutions littéraires, tout en dénonçant les dangers de la littérature.

\section{La métalepse libertaire}

Né à Metz en 1701, Mouhy se dessine comme une personnalité balzacienne avant l'heure : romancier, journaliste, gazetier, mais aussi espion et mouche au service du général de Police, Hérauld, il écrit sur des milliers de pages les aventures d'enfant enlevé (Mémoires d'Anne-Marie de Moras, 1739), de Paysanne parvenue (1735), de prisonnier embastillé (Le masque de fer, 1747), de fermier général vertueux (Le financier, 1755), d'espion de police psychologue (La mouche, 1742), et d'autres héros bariolés. Ses détracteurs parlent d'un opportuniste qui écrit avec négligence et désinvolture pour arrondir ses fins de mois; ses admirateurs, qui s'intéressent à ses audaces littéraires, aux mélanges des genres et à ses talents de conteur, ne ratent aucune de ses parutions. Méprisé par la critique littéraire, lu et réédité, Mouhy revendique une liberté qui n'est pas sans rapport avec une vision du monde hétéroclite à l'image de l'actualité bruyante et mouvementée du Paris des Lumières fréquemment mis en scène dans ses romans.

Polygraphe increvable, écrivain prolixe, Mouhy est reconnu à l'unanimité comme l'«auteur le plus fécond du siècle» (Voisenon, Wald Lasowski 2003 : 23). Dans son œuvre, les frontières du réel et de l'imaginaire s'estompent souvent et parfois même les personnages et leurs auteurs se rencontrent à la sortie de l'Opéra pour se chamailler ! (Démeslé survenu à la sortie de l'opéra entre le Paysan parvenu et la Paysanne parvenue, 1735). Mais l'usage fréquent de métalepses ontologiques par le biais desquelles des mondes différents se confondent ne prend plus sa source dans une distanciation critique par rapport à la fiction. Il découle plutôt d'affinités profondes avec un imaginaire baroque fait de violences et de contrastes encore présent chez Prévost et Marivaux. La pratique métaleptique souligne chez Mouhy l'image d'un romancier ludique indifférent aux règles classiques et ouvert aux expérimentations les plus extravagantes. Dans La Mouche (1736), l'auteur surgit dans le récit fictionnel que constitue l'histoire du manuscrit trouvé pour parler de son public : «Je ne puis l'obliger à me croire; rien n'est plus libre que la façon de penser : et si j'étais à sa place, j'en dirai peut-être davantage " (Mouhy 1736, Herman 1991: 177). La métalepse fournit ici l'alibi nécessaire pour se libérer de toute obligation envers un lecteur prévenu qui, en retour, se libère du joug auctorial et peut se permettre de croire ce qu'il veut, comme il veut. Dès lors que, de part et d'autre du contrat de lecture, la supercherie romanesque est dévoilée, le texte atteint une autonomie, il s'affranchit de toute référentialité et est travaillé par un vaet-vient entre la fiction et la réalité.

L'exemple le plus frappant se trouve dans Lamekis ou Les voyages extraordinaires d'un Egyptien dans la terre intérieure avec la découverte de l'île des Sylphides (1735-38). Dans ce long roman truffé d'aventures fantastiques dans les profondeurs de la terre et les hauteurs des cieux, peuplées de monstres, de sylphes et autres créatures prodigieuses, la métalepse la plus extraordinaire se situe entre les deux parties du récit. Au milieu du livre, un épisode étrange a lieu. L'auteur nommé Mouhy apparaît dans le monde fictionnel pour raconter l'invasion des personnages de son roman dans son univers réel. Il s'introduit dans le récit enchâssé du philosophe Déhalal, congédie brutalement 
le narrateur éponyme Lamekis et occupe la tribune pour avouer l'impossibilité de continuer l'histoire de Déhalal, faute de retrouver la suite du manuscrit :

La quatrième partie finit dans cet endroit, et dans la cinquième il ne se trouve aucune trace de l'histoire de Déhalal, ce qui m'ayant fait imaginer que ce défaut venait d'une lacune considérable, ou de la perte de quelques pages de manuscrit; j'ai cru devoir y suppléer en cherchant dans les auteurs les plus savants quelques passages qui pussent m'aider à finir une histoire si intéressante ; deux ans se sont passés à feuilleter dans les bibliothèques les plus connues, tous les savants qui ont écrit dans ce genre, et surtout ceux qui ont commenté les aventures de Lamékis. Je commençais à me rebuter de tant de soins inutiles, lorsqu'une aventure extraordinaire qui mérite d'être rapportée, m'a mis enfin en état d'achever cet ouvrage. (Mouhy, 340).

Certes, en entrant dans la fiction, l'instance littéraire se transforme immédiatement en instance narrative participant à la diégèse (Linvelt 1989). Néanmoins, l'irruption de la voix de l'auteur provoque objectivement l'arrêt de la narration des aventures de Lamékis, propulse le lecteur dans l'espace spatio-temporel de l'écrivain, le Paris du $18^{\mathrm{e}}$ siècle, et l'oblige à écouter un autre récit, celui de l'écriture de l'histoire de Lamékis. L'intrusion métaleptique qui renvoie à la personne de l'auteur permet de mettre en valeur la mainmise de l'écrivain sur la fiction qu'il crée. Pourtant, le récit de " Mouhy au travail» devient rapidement l'histoire fantastique d'un écrivain hanté par ses personnages, puis, progressivement, soumis à leur pouvoir. Il doit invoquer leur aide pour commencer son récit: "grand Déhalal, célèbre Lamékis, qui jouissez encore aujourd'hui de l'existence humaine ! Après tant de siècles, échauffez mon esprit de vos rayons immortels : c'en est fait, je suis exaucé ; l'étincelle a jailli, je me sens embrasé : commençons » (ibid. : 346). Plus loin, ce sont les personnages de papier qui affrontent l'auteur pour lui demander des comptes, le critiquer et exiger de lui des changements. Dans un dernier temps, ils lui dictent littéralement la suite du récit :

Une de mes plumes s'éleva de dedans mon écritoire, comme une aiguille enlevée par l'aimant, plongea son bec dans l'encrier, et puis se mit à écrire naturellement sur du papier préparé à continuer l'ouvrage après lequel j'étais, il me sembla, aux mouvements de cette plume, qu'elle traçait des caractères français, je devinai même quelques mots à ses mouvements; il me parut aussi que l'écriture ressemblait à la mienne, et je ne me trompais pas (ibid. : 383).

Dans cette mise en scène troublante de la folie de l'écriture romanesque, topos souligné en caractères gras par l'auteur-narrateur : « je deviendrais fol, me disais-je, je n'en suis pas éloigné ", "il faut cesser un travail si abstrait, mon cerveau s'échauffe et se tournera à la fin en délire » (ibid. : 358), « le travail a dérouté ma cervelle. Eh bien, j’y renonce absolument, trop heureux s'il est encore tems!» (Ibid.: 361), l'intention de transgresser les frontières qui séparent le réel du fictif est motivée par la fascination qu'exerce le monde imaginaire sur la réalité (Brunet 2008; Bokobza Kahan 2004). Ce n'est plus le réel qui surgit dans la fiction, mais bien la fiction qui envahit l'univers réel et qui le soumet à son irrationalité. L'auteur dans le roman qui est à même de définir la position d'un évènement par rapport à la frontière entre ce qui existe et ce qui est inventé, avoue son incapacité à trancher. Le coup de force que représente l'irruption de l'auteur dans la diégèse est à double tranchant. S'il révèle l'autorité du créateur, il ouvre néanmoins une brèche permettant aux personnages d'échapper à leur cadre et de devenir autonomes.

L'auteur réel met en place un dispositif subtil permettant de superposer des images de soi dans le récit. L'objectif de l'opération consiste à montrer l'identification profonde 
entre l'auteur responsable du texte et son double dans le discours fictionnel. D'une part, se dégage l'ethos du narrateur premier, instance responsable du récit qui s'est laissé complètement engloutir par la masse de récits enchâssés et de narrateurs seconds. La coupure métaleptique, loin de restituer la maîtrise du récit au narrateur, déclenche au contraire l'invasion des personnages dans son monde. D'autre part, il convient de distinguer ce narrateur-auteur perdu et délirant de l'auteur implicite qui, lui, gère avec brio ce chaos narratif. Or ces deux ethè renvoient à l'image de soi de Mouhy dans le champ littéraire. En mettant en scène son moi écrivain otage de ses inventions mais aussi créateur tout puissant, Mouhy se présente comme un romancier passionné et ludique à l'imagination débordante. Un positionnement qu'il affiche inlassablement tant dans ses péritextes adressés au public et à la critique que dans ses romans, réceptacles malléables qui se prêtent aux expérimentations narratives et discursives les plus étonnantes. Mouhy ne se contente pas d'entrainer son lecteur dans l'univers du fantastique à l'aide de la métalepse qui opère un mélange irrationnel de deux mondes distincts, logiquement incompatibles. Il lâche la bride à son imagination et revendique une liberté créatrice qui sape l'idée d'une maîtrise totale et d'une compréhension parfaite du monde.

Cette attitude qui impose une limite au savoir n'est pas exceptionnelle en ce siècle des Lumières (Bokobza Kahan 2006). Elle détermine des pratiques d'écriture transgressives qui anticipent souvent l'idée postmoderne d'une identité multiple et instable. Mouvance qu'affectionne particulièrement Mouhy dans la majorité de ses romans et qui correspond à la fois à une écriture romanesque rocambolesque, souvent échevelée, et à une personnalité ondoyante aux mille visages.

\section{Conclusion}

La métalepse constitue un outil méthodologique important à l'aide duquel se dégage la complexité de l'ethos dans la fiction. Elle montre combien il est difficile de saisir dans le discours de fiction l'ethos de l'auteur, réfractaire à toute définition claire et univoque. Par ailleurs, les concepts d'auteur implicite et de narrateur non fiable évoqués au début de notre réflexion permettent de procéder à une analyse des structures narratives dans les œuvres choisies et de dégager ainsi les nuances distinctives et les strates multiples du récit. Ces notions nous rappellent que le texte de fiction n'accueille jamais la parole directe de son producteur hormis sans doute dans le roman à thèse, exception qui confirme la règle. La fiction n'est ni le reflet d'une vie réelle ni l'incarnation d'une idéologie philosophique, politique ou sociale. Elle ne prétend pas l'être même lorsque les contenus diégétiques ressemblent étrangement à des éléments biographiques ou historiques connus du lecteur. Ce sont les pratiques d'écriture et les procédés discursifs constitutifs de la fiction qui, en creusant l'écart entre la parole de l'auteur implicite et celle du narrateur non fiable, participent de la construction d'un ethos discursif dans le texte. Un ethos qui doit être mis dans un rapport de réciprocité (complémentaire, conflictuelle, asymétrique) avec celui qui se construit dans le champ littéraire déterminé entre autres par les positionnements et les postures de l'auteur au fil de sa carrière.

Enfin, le choix des exemples provenant tous d'une période qui marque la naissance du roman moderne trouve sa motivation dans notre volonté de réparer une injustice. Les études sur la métalepse dans la fiction concernent généralement la littérature post- 
révolutionnaire et contemporaine. Montrer son rôle central et diversifié dans des œuvres moins étudiées sous cet angle, sinon presque inconnues, témoigne de l'importance du procédé narratif pour une analyse de l'ethos et ses significations dans l'œuvre de fiction.

En bref, mon interrogation théorique sur des notions clé empruntées conjointement à l'analyse du discours et à la narratologie permet d'étayer l'idée que toute compréhension d'un texte de fiction repose sur un rapport dynamique entre ce qui se construit dans le discours et l'image d'auteur dans le champ littéraire d'où émerge son œuvre.

\section{BIBLIOGRAPHIE}

Amossy, Ruth. 2006 [2000]. L'Argumentation dans le discours (Paris : Colin)

Amossy, Ruth (éd.). 1999. Images de soi dans le discours. La construction de l'ethos (Genève : Delachaux et Niestlé)

Bokobza Kahan, Michèle. 2009. «L'Auteur dans la fiction : inscription de la marginalité dans les notes ", Bahier-Porte, Christelle et Régine Jomand Baudry (éds). Ecrire en mineur (Paris :

Desjonquères), 51-63

Bokobza Kahan, Michèle. 2006. «Statut du sujet et construction de l'image de soi dans l'œuvre de Dulaurens », Duranton, Henri (éd.), Le pauvre diable. Destins de l'homme de lettres au $18^{e}$ siècle (SaintEtienne : Presses Universitaire de Saint-Etienne), 225-235

Bokobza Kahan, Michèle. 2004. «Intrusions d'auteur et ingérences de personnages : la métalepse dans les romans de Bordelon et de Mouhy », Eighteenth Century Fiction 16 : 4, 639-654

Booth, C. Wayne. 1952. "The Self-Conscious Narrator in Comic Fiction before Tristam Shandy ", PMLA $67: 2,163-185$

Booth, C. Wayne. 1961. The Rhetoric of Fiction (Chicago : Chicago University Press)

Booth, C. Wayne. 2005. «Resurrection of the Implied Author : Why Bother? », Phelan, James \& Peter. J. Rabinowitz (éds). A Companion to Narrative Theory (Oxford : Blackwell), 75-88

Bordelon. 1789. Histoire de Monsieur Oufle (= Voyages imaginaires, songes, visions et romans cabalistiques 36)(Amsterdam : s. éd.), tome 36

Briosi, Sandro. 1986. « La narratologie et la question de l'auteur », Poétique 68, 507-519

Brunet, Mathieu. 2008. L'appel du monstrueux. Pensées et poétiques du désordre en France au 18e siècle (Louvain \& Paris : Peeters)

Burke, Sean. 1992. The Death and the Return of the Author. Criticism and Subjectivity in Barthes, Foucault and Derrida (Edinburgh : Edinburgh UP)

Chatman, Seymour. 1990. Coming to terms, the rhetoric of narrative in fiction and film (Ithaca and London : Cornell University Press)

Coulet, Henri, 1991 [1967], Le roman jusqu'à la Révolution (Paris : Colin) 
Herman, David. 1997. «Toward a Formal Description of Narrative Metalepsis ", Journal of Literary Semantics, $26: 2,132-152$

Furetière, Antoine. 1666. Le roman bourgeois (Paris : Iolly) [rééd. Paris : Le Club du meilleur livre, s. d., exempl. $\left.n^{\circ} 3891\right]$

Genette, Gérard. 1972. Figure III (Paris : Seuil)

Genette, Gérard. 2004. Métalepse. De la figure à la fiction (Paris : Seuil)

Ginsburg Ruth \& Shlomith Rimmon-Kenan. 1999. «Is There a Life after Death? Theorizing Authors and Reading Jazz », Herman David (ed.), Narratologies : New Perspectives on Narrative Analysis

(Columbus : Ohio State UP), 66-87

Linvelt, Jaap. 1989. Essai de typologie narrative (Paris : Corti)

Lanser, S. Susan. 2001. «(Im)plying the Author », Narrative $9: 2,153-160$

Maingueneau, Dominique. 2004. Le discours littéraire. Paratopie et scène d'énonciation (Paris : Colin)

Malina, Debra. 2002. Breaking the Frame. Metalepsis and the Construction of the Subject (Columbus :

Ohio State UP)

Mouhy, Charles de Fieu. 1788. Lamekis ou les voyages extraordinaires d'un Egyptien dans la terre intérieure avec la découverte de l'île des Sylphides (=Voyages imaginaires, songes, visions et romans cabalistiques 20(Amsterdam \& Paris : Cuchet)

Nelles, William. 1997. Frameworks : Narrative Levels and Embedded Narrative (New-York : Peter Lang)

Nünning, F. Ansgar. 2005. « Reconceptualizing Unreliable Narration: Synthesizing Cognitive and Rhetorical Approaches », Phelan, James \& Peter. J. Rabinowitz (éds). A Companion to Narrative Theory (Oxford : Blackwell), 89-107

Pier, John \& Jean-Marie Schaeffer (éds). 2005. Métalepses, Entorses au pacte de la représentation (Paris : Ecole des Hautes Etudes en Sciences Sociales)

Rivara, Annie. 1993. Introduction à Mouhy, Le masque de fer (Paris : Desjonquères)

Sermain, Jean-Paul. 2002. Métafictions (1670-1730). La réflexivité dans la littérature d'imagination (Paris : Champion)

Wagner, Frank, 2002, « Glissements et déphasages. Note sur la métalepse narrative », Poétique 130, 235-253

Wald Lasowski, Patrick. 2003. Le Traité des mouches secrètes (Paris : Gallimard)

Yacobi, Tamar, 2001. « Package Deals in Fictional Narrative : The Case of the Narrator's (Un)

Reliability", Narrative. $9: 2,223-229$

\section{RÉSUMÉS}

La perméabilité des frontières entre l'intérieur et l'extérieur du texte de fiction nécessite la conciliation des concepts narratologiques d'« auteur implicite » et de «narrateur non fiable » qui privilégient la fonction interne du texte avec la notion d' « ethos discursif » qui ouvre le texte en amont, versant auteur. Le concept clé de l'article - la «métalepse» - comprise ici comme l'intrusion d'un niveau narratif dans un autre, souligne dans le récit la spécificité de la relation causale qui unit l'auteur à son œuvre. La combinaison de ces outils méthodologiques vise à 
proposer une piste de travail permettant de dégager l'ethos auctorial toujours fuyant dans un discours fictionnel.

The permeability of the borders between the inside and the outside of fiction invites us to combine narratological concepts, as "implied author" and "unreliable narrator" concentrated on the internal mechanisms of the text, and the concept of "discursive ethos" opening the text toward its external source, the author. The key concept of this article - the "metalepsis" - defined here as the intrusion of one narrative level into another - emphasizes the special relationship between the author and his work in the narrative. The combination of these methodological tools offers an analytical approach facilitating the apprehension of the unstable auctorial ethos in the fictional text.

\section{INDEX}

Mots-clés : auteur, auteur implicite, ethos auctorial, fiction, image de soi, métalepse, narrateur non fiable

Keywords : auctorial ethos, author, fiction, implied author, metalepsis, self-image, unreliable narrator

\section{AUTEUR}

\section{MICHĖLE BOKOBZA KAHAN}

Université de Tel-Aviv, Département de Français, ADARR 